\title{
El concepto de lo innato en la psicología evolucionista*
}

\author{
Claudia LORENA GARCÍA \\ Instituto de Investigaciones Filosóficas \\ Universidad Nacional Autónoma de México \\ clga@servidor.unam.mx
}

Resumen: Se revisa aquí la literatura correspondiente a algunas disciplinas cognosciti-
vas evolucionistas, y se muestra que existe por lo menos una connotación importante
asociada al término "innato" como se usa en estas disciplinas; a saber, la que entiende
lo innato como aquello que es producto de la evolución por selección natural. Después
se examinan tres propuestas recientes que caracterizan lo innato usando conceptos e
ideas de la biología y se concluye que, aun cuando las caracterizaciones de lo innato
que proponen André Ariew y William Wimsatt recogen aquella connotación evolucio-
nista, éstas deben rechazarse, puesto que tienen problemas conceptuales serios o bien
son incompatibles con otras connotaciones importantes de lo innato en esas disciplinas
cognoscitivas evolucionistas. Se expone que la tercera propuesta -la propia- no tiene
los problemas de las anteriores y sí muchas de sus ventajas. Palabras clave: ciencias cognoscitivas, biología, canalización, atrincheramiento generativo

\begin{abstract}
Some of the literature pertaining to certain evolutionary cognitive disciplines is here reviewed.It is shown that there is at least one important connotation associated to the term "innate" as it is used in those disciplines; to wit, that an innate feature is one which is the product of evolution by natural selection. Then three recent proposals are examined characterizing innateness through concepts and ideas from biology -i.e., André Ariew's, William Wimsatt's and mine- and conclude that, although Ariew's and Wimsatt's proposals capture the aforementioned evolutionary connotation, they must be rejected since they either have serious internal problems, or else are incompatible with other important connotations associated to the term innate in evolutionary cognitive disciplines. It is also shown in this paper that my proposal does not share the problems that the previous ones had, but does share some of their advantages.
\end{abstract}

Key words: cognitive sciences, biology, canalization, generative entrenchment

\section{Introducción}

En la literatura contemporánea de aquellas disciplinas que buscan construir explicaciones evolucionistas de fenómenos cognoscitivos y conductuales tanto en los animales como en los seres humanos, el término "innato" tiene diversas connotaciones y aparece relacionado con diferentes criterios empíricos y teóricos. Aquí examinaré algunas propuestas recientes que pretenden caracterizar una noción coherente y teóricamente útil de lo innato

*Agradezco al dictaminador de esta revista sus valiosas sugerencias a una versión anterior del presente ensayo, y a Maite Ezcurdia y Sergio Martínez por haberse tomado la molestia de leer con cuidado el texto y hacerme comentarios. 
mediante conceptos e ideas de la biología del desarrollo o de la biología evolucionista (o ambas), y presentaré mi propia propuesta. Para evaluar estas propuestas se utilizará el siguiente criterio: la propuesta debe capturar en forma coherente y explicar de maneras biológicamente interesantes algunas de las connotaciones más importantes asociadas al término "innato" tal y como éste se usa en las disciplinas cognoscitivas evolucionistas consideradas y, en especial, debe capturar lo que llamaré la "connotación evolucionista adaptacionista" que es, como veremos, central para el concepto de lo innato en esas disciplinas cognoscitivas.

A mi insistencia de que una caracterización aceptable de lo innato explique de maneras biológicamente interesantes la mayoría de las connotaciones de lo innato en las disciplinas cognoscitivas que aquí consideraremos, le subyacen tres presuposiciones que no argumentaré aquí: (a) la primera -que comparto con todos los estudiosos de fenómenos cognoscitivos y conductuales desde una perspectiva biológico-evolucionista- es que las explicaciones del desarrollo y de las características de las capacidades cognoscitivas — por lo menos las básicas - de los animales y los humanos tarde o temprano tendrán que exhibir una continuidad sustancial (no meramente una analogía o una similitud superficial) con las explicaciones de otros rasgos fenotípicos no cognoscitivos pertenecientes a la biología evolucionista y del desarrollo; (b) que siendo la noción de lo innato históricamente una noción causal que caracteriza ciertos rasgos (estados, capacidades, etcétera) en términos del tipo de causas que esos rasgos tienen, la etiología de esos rasgos por tanto debe ser importante en una caracterización de lo innato, y dado que la etiología de por lo menos algunos rasgos cognoscitivos tendrá que ver, tarde o temprano, con su biología, se sigue que una caracterización de lo innato aceptable para los propósitos de las disciplinas cognoscitivas evolucionistas tendrá que hacer referencia (o por lo menos indicar vagamente) a la etiología biológica de un rasgo innato, y (c) que una noción aceptable de lo innato tiene que ser aplicable en principio a todos los rasgos fenotípicos, cognoscitivos o no, de los organismos biológicos relevantes y no únicamente a sus rasgos cognoscitivos. Adopto esta tercera presuposición por la siguiente razón: aunque en el siglo XVII $-\mathrm{y}$ por razones que expongo en otra parte- ${ }^{1}$ se aplica el término "innato" únicamente a capacidades, disposiciones y estados mentales humanos, el término tiene entonces por lo menos un sentido más general y causal tal que, más adelante, el término puede aplicarse de manera coherente a los rasgos biológicos no cognoscitivos de otros organismos. Creo que es importante rescatar esta parte de la historia del concepto de lo innato al proponer una explicación (explication) de ese concepto que

\footnotetext{
${ }^{1}$ Véase mi "The Concept of Innateness in Rationalists and Empiricists in the Seventeenth Century", inédito.
} 
les permita usarlo tanto a los biólogos como a los psicólogos contemporáneos. $^{2}$

Procederé de la siguiente manera: primero analizaré la manera en que algunos etólogos cognoscitivos, psicólogos evolucionistas y psicólogos del desarrollo con inclinaciones evolucionistas usan el término "innato", y mostraré que la connotación evolucionista adaptacionista está presente en su uso. Mostraré también que, en esos textos, existe una segunda connotación de lo innato - a saber, la de lo innato como lo no aprendido- que no tiene conexión conceptual alguna con la connotación evolucionista adaptacionista y que no cumple con el requisito antes mencionado de que un concepto aceptable de lo innato sea aplicable en principio a todos los rasgos fenotípicos, cognoscitivos o no, de los organismos biológicos relevantes y no únicamente a sus rasgos cognoscitivos.

Después examinaré tres propuestas recientes que pretenden caracterizar de manera precisa una noción de lo innato mediante algunos conceptos de diferentes ramas de la biología, principalmente de la biología evolucionista y la biología del desarrollo. Mostraré que la primera propuesta - que caracteriza lo innato usando el concepto biológico de canalización- sí parece recoger la connotación evolucionista adaptacionista, pero tiene serios problemas conceptuales de coherencia interna y no existe una manera adecuada de resolver estos problemas y salvar la propuesta. Adicionalmente, mostraré que, aun cuando las otras dos propuestas - la que caracteriza lo innato en términos de la noción de atrincheramiento generativo y la mía, que lo caracteriza usando un concepto de factor causal típico de una población - incorporan de alguna manera la connotación evolucionista adaptacionista, la tercera (es decir, mi propuesta) es preferible puesto que recoge de manera más directa y precisa la connotación en cuestión, amén de ser mucho más adecuada para otras connotaciones importantes asociadas al concepto de lo innato en las disciplinas cognoscitivas evolucionistas que aquí considero. ${ }^{3}$

\footnotetext{
${ }^{2}$ Véase también la nota 24 , infra.

${ }^{3}$ En García 2003 planteo una propuesta diferente, pero compatible con la que aquí presento acerca de lo innato. Ahí sostengo que la palabra "innato" debería eliminarse de la mayoría de los contextos teóricos y ordinarios en los cuales se usa, pues en esos contextos conlleva una connotación muy fuerte, que yo llamo "internalista", de acuerdo con la cual lo innato es aquello cuyas causas todas son "internas" del organismo. Argumento que, dados algunos descubrimientos relativamente recientes de la biología del desarrollo, es muy probable que no exista ningún rasgo o característica de ningún organismo que sea innato en este sentido internalista. Sin embargo, en el presente artículo argumento algo diferente: que en algunas disciplinas cognoscitivas de corte evolucionista la palabra "innato" tiene por lo menos una connotación que es teóricamente interesante y que merece ser incluida en una explicación de lo innato que cumpla con ciertos requisitos teóricos relativamente estrictos, i.e. coherencia, adecuación, fertilidad teórica, etc. El argumento que aquí desarrollo se presenta también en García 2004, donde, no obstante, pongo énfasis en partes del argumento distintas de las que destaco aquí; por ejemplo, allá subrayo la parte más abstracta de la construcción conceptual
} 


\section{La psicología evolucionista}

En esta sección caracterizaré primero de manera muy general los presupuestos teóricos que comparten la mayoría de los científicos que trabajan en aquéllas áreas de la psicología cognoscitiva clásica —es decir, no conectivista- en las que se busca describir y explicar el aprendizaje y la conducta en los animales y en los humanos desde una perspectiva evolutivo-adaptativa. Después analizaré los contextos en los que aparecen algunas de sus afirmaciones innatistas y las connotaciones centrales asociadas al concepto de lo innato en estas disciplinas. Aquí consideraré como perteneciente al ámbito de la psicología evolucionista todo estudio del desarrollo y la estructura cognoscitivos y de la conducta de cualquier organismo biológico que comparta los dos supuestos antes mencionados: el supuesto de la psicología cognoscitiva clásica y el de la evolución por selección natural. Así, lo que yo llamaré "psicología evolucionista" incluye una variedad de estudios y de enfoques relativamente distintos de la conducta y el desarrollo cognoscitivo en animales y en humanos. Aquí me interesa indagar si existe un núcleo compartido de connotaciones asociadas al término "innato" en esta diversidad de estudios.

\subsection{Psicología, evolución y función}

El supuesto fundamental de lo que llamaré la "psicología cognoscitiva clásica", de la cual la psicología evolucionista es una vertiente, es la idea de que la mente es una computadora -0 , por lo menos, es similar a una-, que consiste en una serie de mecanismos que procesan información almacenada en una "memoria". Así, para explicar una determinada conducta, según la psicología clásica tenemos que encontrar el mecanismo o la serie de mecanismos psicológicos que la produjeron, así como su estructura, identificando principalmente las funciones psicológicas que llevan a cabo esos mecanismos. Una función psicológica se entiende como la tarea específica de procesamiento de información que ese mecanismo lleva a cabo; por ejemplo, cierto tipo de inferencias, procesamiento de información visual o lingüística, etc. Además, un psicólogo clásico interesado en el estudio del desarrollo cognoscitivo de un determinado organismo - que desee indagar cómo desarrolla ese organismo ciertas capacidades y/o habilidades cognoscitivas- primero tendrá que identificar las funciones psicológicas de esas capacidades o habilidades a fin de poder explicar su desarrollo.

A más del supuesto anterior, los psicólogos evolucionistas comparten un supuesto adicional: que nuestras capacidades cognoscitivas tienen funcio-

del argumento, mientras que aquí remarco más las connotaciones concretas asociadas a la palabra "innato" en la psicología evolucionista contemporánea y la adecuación de mi propuesta a esas connotaciones. 
nes adaptativas, es decir, propiedades funcionales que fueron adquiridas como consecuencia de la evolución por selección natural. ${ }^{4}$ Así, los psicólogos evolucionistas añaden a la noción de función psicológica la idea de que la mayoría de los mecanismos cognoscitivos que subyacen en la conducta de un organismo, si no es que todos, tienen funciones adaptativas ${ }^{5} \mathrm{y}$, por ende, que la mejor manera de descubrir las funciones psicológicas de las capacidades cognoscitivas de un organismo es la de descubrir sus funciones adaptativas. $^{6}$

Muchos psicólogos evolucionistas añaden una afirmación más a los supuestos que consideran fundamentales de un enfoque evolucionista adaptacionista para el estudio de las capacidades cognoscitivas en los animales y los humanos; a saber, la idea de que la mayoría de éstas son capacidades especializadas para llevar a cabo tareas específicas de procesamiento de información de dominios específicos, y no procesadores de información generales que admitan como insumos tipos de información de dominios diversos, por ejemplo, de todas las modalidades sensoriales, o de ámbitos proposicionales de todo tipo. ${ }^{7}$

Por ejemplo, C.R. Gallistel, Ann L. Brown, Susan Carey, Rochel Gelman y Frank C. Keil, en su "Lessons from Animal Learning for the Study of Cognitive Development" (Gallistel et al. 1991, pp. 3-36), intentan extraer algunas lecciones del trabajo de los etólogos, los cuales, según ellos, "buscaron descubrir la diversidad de soluciones animales a presiones ambientales diferentes (o idénticas) [...]; la tesis principal de este credo es el aprendizaje dirigido innata o preferencialmente [...], lo que re-

${ }^{4}$ Cosmides y Tooby afirman: "Un enfoque adaptacionista de la psicología conlleva la búsqueda de un diseño adaptativo, lo que implica entender cómo es que ciertos rasgos del diseño constituyen una maquinaria orgánica diseñada por la selección para resolver un problema. Tal unidad funcional o mecanismo es una adaptación" (Cosmides y Tooby 1997, p. 86; en general, las traducciones de las citas son mías).

${ }^{5}$ Diferentes psicólogos evolucionistas ponen un énfasis diferente en este supuesto central. Algunos — como Leda Cosmides, John Tooby y Dan Sperber - tienden a pensar que todas o casi todas nuestras capacidades cognoscitivas evolucionaron de esta manera, mientras que otros psicólogos evolucionistas son más cautelosos en sus afirmaciones a este respecto. Véase la siguiente nota, y Sperber 1994, pp. 42-46. Otro psicólogo que acepta el supuesto evolucionista en una versión radical es Gerd Gigerenzer (véase 2001, pp. 37-50).

${ }^{6}$ En general, los psicólogos evolucionistas aceptan que las funciones adaptativas y las funciones psicológicas de un mismo mecanismo cognoscitivo pueden ser distintas. Sperber, por ejemplo, concede que la función de un mecanismo visto como un producto de la selección natural (y su dominio "propio") puede ser distinta de la función psicológica que actualmente cumple ese mecanismo (y su dominio "actual”). Véase Sperber 1994, pp. 51-52.

${ }^{7}$ Por ejemplo, Sperber defiende de manera interesante la idea de que el supuesto de la especificidad de dominio es fundamental en un enfoque evolucionista (véase Sperber, 1994, pp. 42-46); Cosmides y Tooby también defienden esta idea (1997, p. 93, y 1998, pp. 198199), lo mismo que Sara J. Shettleworth (1998, p. 44). En García 2005 caracterizo de manera más precisa la noción de especificidad de dominio que se maneja en la psicología de la inferencia humana. 
fleja la presión adaptativa selectiva de un nicho (niche) ambiental particular". 8

Hay dos postulados teóricos que subyacen en el trabajo de Gallistel et al. y de la mayoría de los etólogos cognoscitivos: el primero de ellos relaciona lo innato con aquello que tiene alguna ventaja adaptativa; es decir, que si algo es innato, entonces es producto de la evolución por selección natural (y/o viceversa). Llamaré a este postulado connotación evolucionista adaptacionista de lo innato. El segundo postulado afirma que una teoría del aprendizaje que postule un mecanismo cognoscitivo de aprendizaje completamente general, que no es específico de ningún dominio - por ejemplo, un mecanismo puramente asociativo- es insuficiente y/o innecesaria para explicar la mayoría de los casos de aprendizaje animal en los cuales distintos organismos aprenden a hacer algunas cosas (cantar una determinada canción, orientarse por las estrellas, localizar una fuente de sonido, elaborar una estrategia óptima para encontrar comida, reconocer un depredador, etc.). Por el contrario, Gallistel et al. piensan que los etólogos - biólogos capacitados e interesados en aquellas conductas animales que tienen algún sentido evolutivo- han mostrado ya que los mecanismos cognoscitivos que subyacen en estas conductas y las explican de manera satisfactoria son mecanismos específicos de dominio (i.e., tales mecanismos sólo admiten ciertos tipos de insumos), puesto que están "diseñados" para permitir a los organismos reaccionar a un conjunto de circunstancias ambientales específicas (y sólo a éstas). Esto, añaden ellos, tiene sentido si se piensa en términos evolucionistas:

los ejemplos [que damos acerca del aprendizaje animal] apoyan ampliamente la idea de que en la mayoría de los casos de aprendizaje animal debe haber un mecanismo de aprendizaje específico que hace posible esa clase particular de aprendizaje. Todos estos mecanismos de aprendizaje se han formado a través de las eras evolucionarias [over evolutionary time] de manera tal, que la estructura del mecanismo de aprendizaje - la información que procesa y cómo la procesa- refleja la estructura del problema que ha dado forma a su evolución. (Gallistel et al. 1991, p. 13)

P.S. Davies hace explícito de manera clara el razonamiento que apoya estas afirmaciones:

los organismos se enfrentan a las presiones de selección dentro de un ambiente local y específico y, por lo tanto, los rasgos de los organismos son seleccionados en respuesta a demandas locales y específicas. En consecuencia, es razonable

${ }^{8}$ Gallistel et al. 1991, pp. 4; las cursivas son mías. Subrayo estas dos palabras en su texto puesto que, en esta literatura, aparecen juntas con frecuencia, lo cual refleja un presupuesto importante para nuestros propósitos; a saber, la idea de que si un mecanismo evolucionó por selección natural, entonces es innato (o viceversa). 
especular que los mecanismos que constituyen las mentes de los humanos ahora son en gran medida específicos de dominio-“diseñados" para responder a inputs altamente específicos. ${ }^{9}$

En otro contexto he examinado en detalle este argumento y he mostrado que tiene varias presuposiciones que son, por decir lo menos, muy controversiales. ${ }^{10}$ Por obvias limitaciones de espacio, aquí no podré retomar este asunto. En este contexto mi interés se centra únicamente en indagar cuáles son las connotaciones centrales que giran alrededor del concepto de lo innato en la psicología evolucionista.

\subsection{Lo innato y la selección natural}

Ahora bien, una de las ideas clave que comúnmente se asocian a la noción de lo innato en la psicología evolucionista es la siguiente: un mecanismo cognoscitivo tiene una función adaptativa, en el sentido en que por lo menos una de sus funciones es (o fue) permitir al animal interactuar con su ambiente de una manera adaptativamente ventajosa si (y/o sólo si) es innato. Ésta es la connotación evolucionista adaptacionista.

Pero ésta no es la única connotación ligada a lo innato en la psicología evolucionista. Por ejemplo, Gallistel et al. sugieren también que las siguientes características están asociadas a mecanismos cognoscitivos innatos:

a) cuando diferentes animales resuelven el mismo problema adaptativo de manera diferente;

b) cuando existen apareamientos privilegiados de estímulos, o de estímulos y respuestas, $\mathrm{y}$

c) cuando hay periodos críticos específicos de dominio (Gallistel et al. 1991, p. 32).

Ahora bien, está claro que las primeras dos características están directamente relacionadas sólo con la noción de especificidad de dominio. Dado que Gallistel et al. piensan que es probable que un mecanismo específico de dominio sea producto de la evolución por selección natural y que, por tanto, sea innato, entonces para ellos tiene sentido pensar que las características antes mencionadas estén asociadas a lo innato. No obstante - y como mencioné antes- es controversial que exista una conexión sistemática

\footnotetext{
${ }^{9}$ Davies 1996, pp. 447-448. Nótese que Davies mismo rechaza este argumento.

${ }^{10}$ P.S. Davies (1996) y Lawrence Shapiro y William Epstein (Shapiro y Epstein 1998) también examinan esta tesis, y coinciden —en oposición a muchos psicólogos evolucionistas-en que el supuesto de especificidad de dominio no es fundamental en un enfoque evolucionista en la psicología. Véase también García 2005, donde discuto este supuesto y los argumentos de todos estos autores.
} 
entre la especificidad de dominio de un mecanismo y el que ese mecanismo sea resultado de la evolución por selección natural.

Respecto de la tercera característica, en lo que sigue voy a examinar con cierto detalle la idea de que, si existe un periodo crítico en un mecanismo cognoscitivo de aprendizaje, entonces el mecanismo es (o es probable que sea) innato, puesto que esta idea aparece una y otra vez, no sólo en la etología, sino también en la psicología cognoscitiva en general. Por ejemplo, Elissa L. Newport, en su "Contrasting Conceptions of the Critical Period for Language" (Newport 1991) explica que, en ese artículo,

Me concentro en evidencias de otro tipo a favor de las limitaciones innatas sobre el aprendizaje [...]. En particular, describo evidencias a partir de nuestro trabajo relativo a la adquisición del lenguaje humano, comparando miembros de la misma especie que difieren en los periodos de maduración durante los cuales están expuestos a ambientes de aprendizaje [...]. En este caso, la evidencia a favor de las limitaciones innatas o biológicas proviene del hallazgo de que, a medida que estas limitaciones aparentemente desaparecen o se debilitan con la maduración, la capacidad para aprender declina. ${ }^{11}$

Una capacidad para aprender algo tiene un periodo crítico cuando existe un lapso limitado, normalmente en una etapa temprana, en el cual la capacidad para aprender funciona al máximo, después de lo cual parece disminuir o desaparecer. Hay por lo menos dos maneras distintas de conceptuar y explicar lo que sucede cuando una capacidad para aprender muestra un periodo crítico:

A. Lo que sucede es que el mecanismo mismo que subyace en la capacidad se reduce o desaparece, lo cual produce una disminución de la competencia; o bien

B. no es que el mecanismo de aprendizaje decline o desaparezca, lo que sucede es que otros mecanismos cognoscitivos se desarrollan o cambian y ello interfiere con el funcionamiento de aquel mecanismo.

Newport considera que probablemente haya casos en los que la primera explicación sea la correcta, y otros en los cuales sea preferible la segunda. Sea como fuere, en ninguna de estas dos explicaciones se puede aún visualizar la conexión de un periodo crítico con lo innato. Newport misma sugiere algo que podría proporcionarnos esta conexión. Ella está considerando si existe o no un periodo crítico para la adquisición de un lenguaje en los humanos, y si lo hay, cómo se podría explicar. Para responder estas preguntas, ella y sus colaboradores llevaron a cabo una serie de estudios en personas congénitamente sordas - pero normales en el resto de sus

${ }^{11}$ Newport 1991, p. 111; las cursivas son mías. 
capacidades cognoscitivas, sociales, etc.-, cuya primera exposición a un lenguaje (en todos estos casos se trata del Lenguaje de Signos Americano, LSA) se dio en algunos de ellos a partir del nacimiento y en otros incluso hasta después de la pubertad.

Básicamente, los resultados de estos estudios apoyan la hipótesis de que sí existe un periodo crítico para la adquisición de un primer lenguaje en los humanos: los sujetos estudiados que tuvieron una primera exposición a un lenguaje (LSA) desde su nacimiento tenían un manejo excelente del lenguaje, y entre más tardía había sido esa primera exposición, el manejo de distintos aspectos del lenguaje decrecía correlativamente. Pero, ¿̇cómo explicar estos resultados? ¿Cuál de los dos tipos de explicaciones anteriores es el más plausible en este caso?

Newport se inclina por el segundo tipo de explicación: no es tanto que disminuya el mecanismo mismo de aprendizaje del lenguaje, llamémoslo $M_{L}$, sino más bien que, conforme el individuo se va desarrollando, otros mecanismos cognoscitivos que antes no existían o estaban disminuidos o latentes, empiezan a funcionar y a competir por recursos limitados que antes estaban dedicados casi exclusivamente al funcionamiento de $M_{L}$. Dice Newport:

[Turkewitz y Kenny han mostrado que] el desarrollo normal de cada sistema [sensorial] ocurre en el momento en el que hay limitaciones de maduración (maturational) en la competencia entre sistemas y en la complejidad del insumo que recibe cada sistema. Ellos han sugerido que este patrón de desarrollo en el contexto de las limitaciones puede no ser un accidente o un defecto que deba ser superado, sino más bien una ventaja evolutiva. ${ }^{12}$

De manera similar, Newport cree que las limitaciones cognoscitivas de los niños pequeños constituyen más bien una ventaja computacional para el desarrollo exitoso del lenguaje en esos niños - y, por ende, son una ventaja evolutiva-, puesto que, al no tener demasiadas capacidades cognoscitivas a esa edad, los recursos limitados que tienen pueden dedicarse en mayor medida al aprendizaje exitoso y completo de un lenguaje, cosa que no sucede en los adultos, los cuales tienen todas o la mayoría de sus capacidades cognoscitivas completamente operativas, lo cual requiere muchos recursos que de otra manera no podrían dedicarse al desarrollo completo de un lenguaje. Así, los adultos sólo pueden adquirir un primer lenguaje de una manera fragmentaria y deficiente.

Lo que me interesa destacar de la explicación anterior es la sugerencia, por parte de Newport, de que los periodos críticos en las capacidades de aprendizaje de los animales y de los humanos presuntamente constituyen una ventaja adaptativa. Si esta sugerencia es correcta, y si se acepta lo

${ }^{12}$ Newport, 1991, p. 125; las cursivas son mías. 
que llamé la connotación evolucionista adaptacionista, entonces es natural pensar que la presencia de un periodo crítico en una capacidad de aprender indica que ésta es innata, puesto que la presencia del periodo crítico en esa capacidad constituye una ventaja adaptativa. Así, para explicar por qué se asocia la presencia de un periodo crítico con lo innato en la literatura psicológica no es necesario especular que esto se debe a que la noción de especificidad de dominio debe estar asociada a lo innato en esos contextos.

Resumiendo: hay por lo menos cuatro características que comúnmente aparecen asociadas con lo innato en la etología y en la psicología evolucionista. La primera es la de ser el resultado de la evolución por selección natural y, por ende, la de constituir una ventaja adaptativa (connotación evolucionista adaptacionista). Ésta es la que más me interesa destacar. Las dos siguientes - a saber, la existencia de diferentes soluciones en distintos animales al mismo problema adaptativo, y la existencia de pares privilegiados de estímulos y respuestas- parecen estar asociadas con lo innato, porque se piensa que hay una conexión íntima entre la especificidad de dominio de un mecanismo cognoscitivo y el que ese mecanismo sea resultado de la evolución por selección natural y, por ende, sea innato. Quien - como nosotros- cuestione la existencia de esa conexión íntima, cuestionará la idea de que tales características deban de estar asociadas a lo innato. Finalmente, está la presencia de un periodo crítico en una capacidad de aprendizaje. Expliqué que esta característica se asocia a lo innato probablemente porque se piensa que el que una capacidad tenga un periodo crítico constituye una ventaja adaptativa y, por tanto, que la capacidad misma es innata (connotación evolucionista adaptacionista).

\subsection{Lo innato y lo no aprendido}

En el trabajo de muchos psicólogos evolucionistas se aprecia también la presencia de otra connotación de lo innato, de acuerdo con la cual lo innato es aquello que no es aprendido. Esto puede verse claramente, por ejemplo, en el trabajo de Leda Cosmides y John Tooby, quienes con frecuencia hacen afirmaciones como la siguiente:

Para poder aprender, debe haber un mecanismo que causa el aprendizaje. Puesto que el aprendizaje no puede darse en ausencia de un mecanismo que lo cause, el mecanismo que lo causa debe él mismo no ser aprendido, esto es, debe ser innato. Por lo tanto, ciertos mecanismos de aprendizaje deben ser aspectos de una arquitectura que evolucionó [...]. En cierto sentido, los humanos deben de tener mecanismos de aprendizaje innatos o instintos de aprendizaje. ${ }^{13}$

${ }^{13}$ Cosmides y Tooby 1997, p. 92; las cursivas son mías. 
Aquí hay por lo menos dos conexiones conceptuales sugerentes: en primer lugar, la idea de que aquellos aspectos cognoscitivos que son innatos en ciertos organismos deben haber evolucionado en esos organismos como resultado de la selección natural (connotación evolucionista adaptacionista). Hemos visto ya cómo esta conexión innato-adaptativa es recurrente en la literatura psicológica examinada hasta ahora. En segundo lugar, encontramos también la idea de que algo que no es aprendido debe ser innato.

Recientemente han surgido varias propuestas para caracterizar el concepto de lo innato que pretenden recoger directamente esta connotación de lo no aprendido con que se ha usado en la historia de la filosofía y de la psicología, desde por lo menos el siglo XVII hasta nuestros días. De hecho, la conexión conceptual entre lo innato y lo no aprendido surge en este siglo, cuando lo que es "interno" - los recursos causales que pertenecen exclusivamente al sujeto- se vuelve sinónimo de lo "mental". Además, si - como he sostenido en otro contexto- el concepto de lo innato que se maneja en este momento es lo que yo llamo "un concepto internalista causal", entonces podemos apreciar cómo es que serían innatos aquellos rasgos cognoscitivos de un sujeto cuyas historias causales remiten exclusivamente a los recursos cognoscitivos del sujeto, sus recursos "internos". Si también se acepta, como lo hicieron los filósofos de esa época, que algo es aprendido cuando su historia causal se refiere tarde o temprano a algunos factores causales "externos" al individuo (es decir, factores no cognoscitivos), entonces puede apreciarse fácilmente cómo llegaron ellos a pensar que un rasgo cognoscitivo innato es aquel que no es aprendido. ${ }^{14}$

Desafortunadamente, por limitaciones de espacio, aquí no podré examinar esta segunda e interesante conexión que sugiere un concepto de lo innato muy diferente de las caracterizaciones que analizaremos más adelante, caracterizaciones que llamaré desarrollistas evolucionistas. Me gustaría señalar, sin embargo, que todos los intentos relativamente recientes por formular algo parecido a esta connotación de lo innato como lo no aprendido - y aquí tengo en mente la propuesta de Jerry Fodor (1982a), Richard Samuels ${ }^{15}$ y M.A. Khalidi (2002), entre otros- comparten la idea de que

\footnotetext{
${ }^{14}$ Para apreciar lo innato como lo no aprendido, por ejemplo, en John Locke, véase Essay, Bk. I, cap. II, §§ 15 y 23.

${ }^{15}$ Samuels (2002) se inclina a pensar que el único constreñimiento conceptual (aparte de un constreñimiento conceptual puramente negativo) que debe cumplir cualquier caracterización de lo innato es que si un rasgo es innato, la consecuencia será que entonces no es aprendido. Véase Samuels 2002, p. 236. Por supuesto que si éste es el único constreñimiento conceptual, entonces parecería que no son aceptables las explicaciones desarrollistas evolucionistas de lo innato que examinaremos aquí (la de Wimsatt, la de Ariew y la nuestra). Sin embargo, Samuels no da ningún argumento para pensar que ésa es la única connotación importante del concepto de lo innato tal y como se usa en las disciplinas cognoscitivas. Ciertamente, como hemos visto, no es la única connotación asociada a lo innato en esas disciplinas; además, ¿̇por qué pensar que es la más importante? En ausencia de algún argumento, yo supondría que lo
} 
tiene sentido hablar de lo innato únicamente en conexión con rasgos, estados o capacidades cognoscitivos (puesto que no tiene sentido afirmar de un rasgo fenotípico no cognoscitivo que es aprendido, o que no lo es). De lo que he dicho anteriormente, y sobre todo en la introducción, queda claro que en mi opinión esto es falso; en el pasado se ha hablado de rasgos no cognoscitivos innatos en la biología al igual que en muchos otros contextos teóricos; pienso además que no es conveniente tener una caracterización de lo innato que no tenga aplicación, utilidad e interés teórico en la biología. Así, creo que, a menos que se pueda mostrar que esta connotación del término "innato" como lo no aprendido tiene algunas relaciones interesantes con algún concepto desarrollista evolucionista de lo innato, habrá que eliminar la conexión entre lo innato y lo no aprendido si las consideraciones discutidas brevemente en la introducción tienen alguna fuerza, algo que no argumentaré aquí.

Procederé ahora a examinar tres caracterizaciones desarrollistas evolucionistas y a evaluarlas en función de si incorporan o no de manera adecuada la connotación evolucionista adaptacionista de lo innato.

\section{Tres nociones biológicas de lo innato ${ }^{16}$}

En esta parte examinaré tres propuestas que pretenden caracterizar de manera precisa una noción de lo innato usando algunos conceptos de diferentes ramas de la biología, principalmente de la biología evolucionista y la biología del desarrollo.

\subsection{Innatismo y canalización}

Hace unos años, André Ariew propuso caracterizar lo innato recurriendo a la noción de canalización, concebida inicialmente por el biólogo del desarrollo C.H. Waddington. La idea intuitiva que subyace en esta noción es que hay ciertos rasgos fenotípicos de algunos organismos que, durante su desarrollo ontogenético, son relativamente insensibles a ciertos tipos de fluctuaciones ambientales más o menos drásticas, y entre más insensibles sean, más canalizados estarán. La siguiente es una formulación más precisa de la noción de canalización, tal y como la conciben Waddington y Ariew: ${ }^{17}$

que Samuels está intentando es simplemente legislar cómo se debe usar el término "innato" en esos contextos, sin siquiera dar buenas razones que apoyen su pretensión legislativa.

${ }^{16}$ En García 2003 y 2004 se encuentran versiones distintas y más detalladas de algunos fragmentos del material que aparece en esta parte del artículo.

${ }^{17}$ En García 2004 desarrollo en forma detallada un argumento a favor de esta manera de hacer más precisa la noción de canalización, tal como la entienden Waddington y Ariew. Presento aquí unicamente las partes que considero necesarias para evaluar la propuesta de Ariew respecto a lo innato en función de su adecuación a la manera como se usa este concepto en las disciplinas cognoscitivas evolucionistas. 
Un determinado proceso de desarrollo que típicamente culmina en un rasgo fenotípico $T$ de un organismo $O$ de población $P$ está canalizado en grado $p(1 \geq p \geq 0)$ en el desarrollo ontogenético de $O$ (respecto de un genotipo o conjunto de genotipos ${ }^{18}$ y respecto de ciertas variables ambientales, $V_{1}, \ldots, V_{n}$ típicas del desarrollo de los miembros de la población $P$ a la que $O$ pertenece y en las que estos miembros desarrollan el rasgo $T$ ) si y sólo si hay un rango amplio de cambios $V_{1_{1}}, \ldots, V_{1_{m}}$ relativos a $V_{1}$, otro rango amplio de cambios $V_{2_{1}}, \ldots, V_{2_{r}}$ relativos a $V_{2}, \mathrm{y}$ un rango amplio de cambios $V_{n_{1}}, \ldots, V_{n_{s}}$ relativos a $V_{n}$, tales que la probabilidad de que el rasgo $T$ de $O$ de todas maneras habría aparecido en $O$ aun cuando para cada variable $V_{i}(i=1, \ldots, n)$, uno de los cambios $V_{i_{j}}$ se hubiera dado, es mayor o igual a $p$.

El problema central con esta noción de canalización se puede visualizar si consideramos el caso en el que hay un rasgo $T_{1}$ que está muy canalizado en un organismo $O_{1}$ relativo a un rango amplio de cambios en la variable ambiental $V_{\alpha}$, pero que está canalizado en un grado muy pequeño respecto de la variable ambiental $V_{\beta}$, mientras que en un organismo $\mathrm{O}_{2}$ es "al revés": un rasgo $T_{2}$ está canalizado en un grado muy pequeño en el desarrollo de $\mathrm{O}_{2}$ respecto de la variable $V_{\alpha}$, pero está muy canalizado respecto a un rango amplio de cambios en $V_{\beta}$. En este caso, la pregunta por responder es la siguiente: ¿Diremos que $T_{1}$ está canalizado en $O_{1}$ en el mismo grado (o en mayor o en menor grado) en el que $T_{2}$ lo está en $\mathrm{O}_{2}$ ? Ahora bien, la manera razonable de responder esta pregunta requeriría saber qué tan amplio es el rango de cambios en $V_{\alpha}$ relativo al cual $T_{1}$ está canalizado en el desarrollo de $O_{1}$, y qué tan amplio es el rango de cambios en $V_{\beta}$ respecto del cual se dice que $T_{2}$ está canalizado en el desarrollo de $\mathrm{O}_{2}$. Sin embargo, aun cuando supiéramos esto, no habría manera de comparar qué tan amplios son los rangos de cambios en $V_{\alpha}$ y $V_{\beta}$ en ambos casos, puesto que estaríamos hablando de dos variables ambientales muy distintas que están sujetas a parámetros de medición inconmensurables; por ejemplo, temperatura o intensidad de la luz o cantidad de una determinada sustancia química en el ambiente intrauterino, etcétera. ${ }^{19}$

\footnotetext{
${ }^{18}$ Como veremos más adelante, debe hacerse la relación con un genotipo (o conjunto de genotipos), ya que con frecuencia sucede que dos genotipos distintos asociados a un mismo fenotipo muestran distintos grados de insensibilidad a diferentes variables ambientales y a diversos rangos de cambios en tales variables.

${ }^{19} \mathrm{El}$ que diferentes rasgos fenotípicos de un organismo (asociados a un mismo genotipo) puedan reaccionar de maneras muy distintas a cambios en la misma variable ambiental durante el desarrollo, y el que diferentes genotipos también muestren diferentes grados de sensibilidad a una variable ambiental con respecto al mismo fenotipo, pueden apreciarse a partir de los siguientes ejemplos: la sensibilidad del peso corporal del adulto a la temperatura durante el desarrollo en Drosophila — la mosca de la fruta- es muy alta, pero el tamaño del
} 
Por lo tanto, la noción de canalización propuesta por Waddington y Ariew es muy heterogénea respecto de los tipos de factores que determinan el grado de canalización de un proceso de desarrollo, tan heterogénea que en principio es imposible la formulación de criterios precisos y generales que permitan determinar el grado de canalización de un rasgo fenotípico en un organismo (con respecto a todas las variables ambientales biológicamente relevantes). Quizá ésta sea la razón por la que muchos biólogos tienden a considerar que la noción de canalización es irremediablemente metafórica y que nunca podrá llegar a ser un concepto que denote una clase de fenómenos teóricamente importante en la biología del desarrollo. ${ }^{20}$

Ahora bien, esta objeción de heterogeneidad podría eludirse si se relaciona el concepto de canalización con una única variable ambiental de la siguiente manera:

Un determinado proceso de desarrollo que típicamente culmina
en un rasgo fenotípico $T$ de un organismo $O$ de población $P$ está
canalizado en grado $p(1 \geq p \geq 0$ ) en el desarrollo ontogenético
de $O$ (respecto de un genotipo o conjunto de genotipos, y res-
pecto de una variable ambiental $V$, típica del desarrollo de los
miembros de la población $P$ a la que $O$ pertenece y en las que
estos miembros desarrollan el rasgo $T$ ) si y sólo si hay un rango
amplio de cambios $V_{1}, \ldots, V_{m}$ relativos a la variable $V$ tales que,
para cada $V_{i}(i=1, \ldots, m)$, la probabilidad de que el rasgo $T$
de $O$ de todas maneras habría aparecido en $O$ aun cuando $V_{i}$
hubiese ocurrido, es mayor o igual a $p^{21}$

ojo compuesto en Drosophila en el genotipo "Wild-type" en el locus Bar no es muy sensible a los cambios en la temperatura durante el desarrollo, mientras que la sensibilidad del tamaño del ojo a las variaciones en la temperatura es diferente para otros genotipos de Drosophila en el locus Bar. Véase Futuyma 1998, p. 41.

${ }^{20}$ Willem Scharloo revisa varios experimentos llevados a cabo por Waddington, y más recientemente por otros biólogos, en Drosophila Melanogaster y en ratones. Aun cuando Scharloo se siente inclinado a aceptar el concepto de canalización, hacia el final de su artículo concluye: "¿Significa esto que tenemos que abandonar el concepto de canalización? Claro que no. Es un hecho que el tipo salvaje (wild-type) [Drosophila] es relativamente constante comparado con los mutantes y las fenocopias [...]. Sin embargo, tenemos que reconocer que nuestra comprensión de este tema fascinante todavía es limitada" (Scharloo 1991, p. 89).

Por otra parte, James McClaurin nos dice que "La explicación de Waddington [de la canalización] nos proporciona una metáfora útil y relevante en relación con la forma en que una multitud de factores constreñidores influyen en el desarrollo biológico. Aun así, sigue siendo una metáfora" (McClaurin 2002, p. 127; las cursivas son mías).

${ }^{21}$ Esta caracterización de la canalización no es aún todo lo precisa que se desearía, ya que probablemente el mismo proceso de desarrollo que culmina en un rasgo fenotípico $T$ muestre distintos grados de sensibilidad a los cambios en una variable ambiental dada, dependiendo de la etapa del desarrollo - temprana o tardía, por ejemplo- en la cual ocurran los cambios en cuestión. Así, es probable que un mismo proceso de desarrollo (respecto de un mismo genotipo y de una misma variable ambiental) esté muy canalizado cuando los cambios en tal 
Sustituyendo "canalizado en grado $p$ " por "innato en grado $p$ " en la anterior caracterización tenemos la propuesta de Ariew relacionada con lo innato. Examinaré ahora esta última propuesta: primero, se podría sostener quizá que, en cierto sentido, la caracterización de lo innato de Ariew sí recoge la connotación evolucionista adaptacionista, puesto que es probable que el que un determinado proceso de desarrollo esté muy canalizado significa que éste es un producto de la selección natural. Waddington, por ejemplo, sostiene que: "Si es una ventaja, como normalmente parece serlo para mecanismos del desarrollo, el que una respuesta obtenga un valor óptimo de manera más o menos independiente de la intensidad del estímulo ambiental, la reactividad se irá canalizando, una vez más, con la influencia de la selección natural" (Waddington 1942, p. 563). Sin embargo, el problema ahora es que un concepto de lo innato como éste, relativizado a una única variable ambiental, se desvía de manera sustancial de la forma en que se usa el término "innato" en los estudios cognoscitivos del aprendizaje y la conducta en animales y humanos desde una perspectiva evolucionista. Por ejemplo, es improbable que cuando un psicólogo del desarrollo afirme que el mecanismo cognoscitivo que permite el aprendizaje del lenguaje en los humanos es innato, lo que quiera sea plantear una afirmación relativizada como la siguiente: que los humanos de todas formas habrían desarrollado ese mecanismo, aun cuando durante su desarrollo ontogenético hubiese ocurrido uno de los cambios en una determinada variable ambiental única, digamos, la intensidad de la gravedad.

Asimismo, si aceptásemos esta noción de lo innato, entonces tendríamos que admitir la posibilidad de que con frecuencia el mismo rasgo asociado al mismo genotipo sea, al mismo tiempo, muy innato respecto de un rango amplio de cambios en una variable ambiental $V$, e innato en un grado muy pequeño (o incluso no innato) con respecto a otro rango de cambios en una variable ambiental distinta $V^{*}$. No obstante, la noción de lo innato, tal y como se usa en las disciplinas cognoscitivas consideradas aquí, nunca se relativiza de esta manera. El etólogo Peter Marler, por ejemplo, jamás ha afirmado que es posible o probable que un determinado rasgo $R$ del canto de los pájaros de una determinada población de una especie sea innato en relación con una cierta variable ambiental $V$ y no innato en relación con otra variable ambiental $V^{*}$, aun cuando Marler sostiene afirmaciones innatistas en todas partes (Marler 1991).

En conclusión, es improbable que una noción de lo innato que esté relacionada con una variable ambiental particular sea la noción a que se apela en las disciplinas cognoscitivas evolucionistas que he considerado aquí.

variable se den en etapas tardías del desarrollo, pero que no esté canalizado (o lo esté muy poco) cuando esos cambios ocurren en etapas tempranas. 


\subsection{Innatismo y atrincheramiento generativo ${ }^{22}$}

Otro importante filósofo de la biología que ha propuesto una caracterización de lo innato recientemente es William Wimsatt, quien usa su noción de atrincheramiento generativo para estos propósitos como sigue (ésta es una primera aproximación): "Un rasgo $T$ de un organismo $O$ de la especie $S$ es innato en $O$ si y sólo si $T$ está generativamente atrincherado en $O$ " (Wimsatt 1986). La idea general que subyace en la noción de atrincheramiento generativo es la siguiente: entre los rasgos fenotípicos que aparecen en determinada etapa del desarrollo ontogenético de un organismo, hay algunos de cuya aparición dependen causalmente más rasgos, los cuales por ende están más atrincherados. Dice Wimsatt: "Diré, por tanto, que ciertos rasgos están 'generativamente atrincherados' en el grado en el cual haya cierto número de rasgos que se desarrollan después y que dependen de aquéllos" (Wimsatt 1986, p. 198). Por razones que explico en detalle en otro contexto, la caracterización del atrincheramiento generativo en Wimsatt puede precisarse de manera adecuada de la siguiente manera (véanse García 2003 y 2004):

Un rasgo $T$ está generativamente atrincherado en un organismo $O$ en grado $n$ cuando $O$ tiene un número $n$ de otros rasgos $T_{1}, \ldots, T_{n}(n \geq 1)$ tales que $T$ fue un factor causal en la aparición de $T_{1}, \ldots, T_{n}$ durante el desarrollo ontogenético de $O$, que tuvo lugar en aquellos ambientes que incluyen todos los factores causales típicos de la población a la que $O$ pertenece con respecto a los rasgos $T_{1}, \ldots, T_{n}$ (los cuales, en consecuencia, fueron adaptativos en algún medio ambiente ancestral).

Y la siguiente es nuestra propuesta para caracterizar la noción de factores causales típicos de una población: ${ }^{23}$

En un tiempo $t$, hay una población $P$ de miembros de una especie $S$. Supongamos que los miembros de $P$ vivieron en algún tiempo anterior, $t-e$, en un ambiente $E$ que tenía un aspecto que constituía un problema adaptativo para los miembros de $P$, un problema para el cual tener un rasgo $T$ era una "solución", y que los miembros de $P$ que tenían $T$ y que vivieron en $E$ en $t-e$ tenían mejores radios de supervivencia y reproducción que

${ }^{22}$ La presentación de la propuesta de Wimsatt 1986 que aquí ofrezco es en esencia —aunque no en detalles_ la misma que aparece en García 2004.

${ }^{23} \mathrm{~A}$ mi parecer, ésta es la manera menos problemática de caracterizar la idea de que hay algo así como una manera típica o normal en la que se desarrollan ontogenéticamente los miembros de una especie o de una población, menos problemática de todas las que he visto en la literatura en filosofía de la biología. Véase mi García 2003, pp. 12-14, para una discusión breve de este tema. 
aquellos que vivieron en $E$ pero que no tenían $T$ en $t-e$. En este caso diremos que todos los factores causales (internos y externos) responsables de la aparición de $T$ en los miembros de $P$ en $t-e$ son típicos de la población $P$ con respecto al rasgo $T$ en $t$.

Es importante notar que, para Wimsatt, tiene sentido preguntarse cuán atrincheradas en el desarrollo ontogenético de un organismo están las condiciones ambientales externas a ese organismo (condiciones tales como la temperatura de incubación o la cantidad de gravedad), y no sólo los que tradicionalmente se consideran los rasgos fenotípicos "propios" de ese organismo. Dice Wimsatt: "En esta perspectiva, es claro que la información adquirida del ambiente puede tener un efecto profundo, si está generativamente atrincherada de manera profunda, en relación con la conducta subsiguiente; en este análisis, si está generativamente atrincherada, es innata." (Wimsatt 1986, p. 200; las cursivas son mías.).

Ahora bien, con respecto a la adecuación de la noción de lo innato que Wimsatt propone, hay dos problemas serios: en primer lugar, vimos ya que Wimsatt está dispuesto a decir que una buena parte del ambiente externo en el que se desarrollan la mayoría de los organismos biológicos es innata en esos organismos. Sin embargo, hasta donde yo sé, ningún psicólogo evolucionista —en el sentido amplio en el que aquí uso "psicología evolucionista"- pensaría que la temperatura de incubación de un huevo o la cantidad de gravedad presente durante el desarrollo podrían ser innatas, o no serlo, en un determinado organismo. El término "innato", para estas condiciones ambientales, simplemente no se aplica.

En segundo lugar, si se aceptase la noción wimsattiana de lo innato, entonces resultaría que no son innatos ninguno de los rasgos fenotípicos de un organismo que son o fueron adaptativos y que tienen algunas causas genéticas, pero que ya no son factores causales en la aparición de ningún otro rasgo fenotípico durante su desarrollo ontogenético. Sin embargo, no es así como se usa el término "innato" en las disciplinas cognoscitivas evolutivas consideradas. Por ejemplo, con frecuencia se afirma en estas disciplinas que determinados rasgos de ciertas conductas en organismos de una determinada población son innatos, aun cuando tales rasgos no figuran como factores causales en la aparición de otros rasgos fenotípicos de esos organismos.

En resumen: si bien la noción de atrincheramiento generativo parece ser una noción de gran fertilidad teórica en la biología evolutiva del desarrollo, la noción de lo innato de Wimsatt tiene problemas serios de adecuación a la noción (o nociones) de lo innato que se usa en la psicología evolucionista. 


\subsection{Innatismo y un ambiente típico}

En esta sección desarrollaré una noción de lo innato que se adecua a por lo menos una de las connotaciones centrales de lo innato que encontramos en la psicología evolucionista, que tiene implicaciones biológicas interesantes, que es teóricamente fértil y que en principio puede usarse tanto en la biología como en la psicología. Además, como veremos, mi concepto de lo innato tiene relaciones interesantes con la noción de lo innato propuesta por Elliot Sober (1999) y con el concepto wimsattiano de atrincheramiento generativo, pero difiere de la caracterización de lo innato de Wimsatt en ciertos puntos cruciales que le permiten evitar los problemas de adecuación que este concepto parece tener.

Ahora bien, la caracterización de lo innato que aquí propongo presupone una noción de factores causales presentes en el desarrollo ontogenético de un organismo que son típicos de la población a la que ese organismo pertenece. Cuando introduje la noción wimsattiana de atrincheramiento, tuve ya la ocasión de presentar mi caracterización de esa noción, a saber:

En un tiempo $t$, hay una población $P$ de miembros de una especie $S$. Supongamos que esta población vivió en algún tiempo anterior, $t-e$, en un ambiente $E$ que tenía un aspecto que constituía un problema adaptativo para los miembros de $P$, un problema para el cual tener un rasgo $T$ era una "solución", y que los miembros de $P$ que tenían $T$ y que vivieron en $E$ en $t-e$ tenían mejores radios de supervivencia y reproducción que aquellos que vivieron en $E$ pero que no tenían $T$ en $t-e$. En este caso diremos que todos los factores causales (internos y externos) responsables de la aparición de $T$ en los miembros de $P$ en $t-e$ son típicos de la población $P$ con respecto al rasgo $T$ en $t$.

Una vez en posesión de esta noción de factores causales tipicos, propongo la siguiente noción de lo innato: ${ }^{24}$

\footnotetext{
${ }^{24}$ En otro contexto propuse una manera de caracterizar la noción de un rasgo fenotípico heredado tal y como se usa en la biología evolutiva (véase García 2003). Más adelante, conforme fui investigando la manera en que se usa la palabra "innato" en la psicología evolucionista (la etología cognoscitiva, la psicología evolucionista, la psicología del desarrollo), descubrí que la propuesta de la noción de un rasgo heredado que yo hacía en aquél contexto se acercaba mucho a por lo menos una de las maneras en que se usa la palabra "innato" en esas disciplinas (y, en particular, a lo que aquí he llamado la connotación evolucionista adaptacionista), lo cual, a mi parecer, resultaba muy interesante y enriquecedor. Mi propuesta de lo innato aquí, por ende, coincide con la noción biológica de herencia que propuse en aquel contexto, aunque aquí el argumento a favor de mi noción de lo innato es muy diferente del argumento a favor de la adecuación de mi noción de herencia en el contexto de la biología evolutiva.
} 
Un rasgo fenotípico $T$ de un organismo perteneciente a una población determinada de una especie es innato en ese organismo en un tiempo $t$ cuando:

(a) El organismo en cuestión posee $T$ en $t, \mathrm{y}$

(b) $T$ apareció en ese organismo como resultado de su desarrollo ontogenético en un ambiente que incluye todos los factores causales típicos de esa población con respecto al rasgo $T$ en $t$, entre los cuales encontramos algunos factores genéticos.

Nótese que, de acuerdo con mi noción de lo innato, un determinado mecanismo cognoscitivo es innato en, digamos, un ser humano si ese mecanismo les dio una ventaja evolutiva a los humanos, ancestros suyos, en los que inicialmente apareció tal mecanismo (en las condiciones ambientales en las que ellos vivían), y además ese mecanismo aparece ahora en aquel ser humano como resultado de la interacción de un número de genes con los factores causales típicos de la población en la cual el mecanismo en cuestión surgió inicialmente. ${ }^{25}$ Por ejemplo, es probable que el mecanismo cognoscitivo que les permite a los humanos conocer la mente de los otros (i.e., "leer la mente" de los otros) ${ }^{26}$ les dio una ventaja adaptativa, en cuyo caso este mecanismo sería innato (en mi sentido) si además su aparición en los humanos se debiera a factores genéticos en ciertas condiciones "típicas" de la población en la que tal mecanismo inicialmente haya aparecido. Sin embargo, la atribución de un tipo particular de creencia a un humano por parte de otro humano probablemente no constituye una ventaja adaptativa para los humanos y no es, por ende, innata. Tampoco sería necesario considerar innata la fijación de recuerdos particulares - aun cuando hay razones para pensar que tal fijación requiere expresión genética (véase Kandel, Schwartz y Jessell 1995, pp. 674, 680 y 685)—, a menos que tal fijación constituya una ventaja adaptativa para los humanos y tenga una historia

\footnotetext{
${ }^{25}$ Puesto que, en este sentido, lo innato se refiere a una población, si un organismo individual se incorporara a una población distinta de la misma especie (una población que, por ejemplo, evolucionó en un ambiente apropiadamente distinto), es posible que los rasgos de ese organismo que antes eran innatos ya no lo sean ahora, o viceversa. Esto se debe a que el que un rasgo sea o no innato, de acuerdo con esta caracterización, está determinado por la historia evolutiva de una población a la cual pertenece el organismo de que se trata. Esta consecuencia indeseable puede evitarse si ajustamos nuestra manera de entender lo que significa que un organismo pertenezca a una determinada población; por ejemplo, imponiendo ciertos requisitos filogenéticos a la pertenencia a una población

${ }^{26}$ Esto es lo que algunos psicólogos de habla inglesa llaman mind-reading capacity, la capacidad de adscribir correctamente creencias, deseos, emociones, intenciones, etc., a los otros.
} 
causal típica (en mi sentido). ${ }^{27}$ Así, que un rasgo cognoscitivo determinado tenga un componente genético no es suficiente - aunque sí necesariopara considerarlo innato.

Otra manera de formular mi propuesta de caracterizar lo innato - una manera que pueda serle útil a algunos teóricos de la biología para entenderla mejor- podría consistir en usar la terminología de "procesos de desarrollo" (developmental processes) que acuñan P.E. Griffiths y R.D. Gray, quienes, paradójicamente, proponen una eliminación total del término "innato" de todas las ciencias naturales, incluyendo la psicología evolucionista. Sin embargo, usando la terminología de estos autores, podría decir que un rasgo es innato cuando es resultado de aquellos recursos causales (genéticos y epigenéticos) que pertenecen a lo que ellos llaman el "proceso de desarrollo" donde "el proceso de desarrollo consiste en una serie de interacciones con los recursos del desarrollo que exhibe una recurrencia apropiadamente estable en el linaje" (Griffiths y Gray 1994, p. 292). Ahora bien, entre los recursos del desarrollo que muestran "una recurrencia apropiadamente estable", pueden distinguirse varios tipos: (1) aquellos cuya recurrencia estable en el linaje se da en parte gracias a la recurrencia estable en el linaje de ciertos tipos de interacciones entre los organismos de la población en cuestión y su ambiente, y (2) aquellos cuya recurrencia estable en el linaje no requiere causalmente la recurrencia estable de tales interacciones. Lo que yo llamo "factores causales típicos" de una población respecto de cierto rasgo fenotípico incluye recursos causales de ambos tipos.

Podemos ahora señalar que existen algunas relaciones interesantes entre la noción wimsattiana de atrincheramiento generativo y mi noción de lo innato: si un rasgo fenotípico de un organismo es innato (en mi sentido), entonces todos los factores causales típicos de la población a la que el organismo pertenece - al igual que los factores genéticos-, responsables de la aparición de ese rasgo en ese organismo durante su desarrollo, estarán generativamente atrincherados en el organismo, puesto que estoy hablando de un rasgo adaptativo. Al mismo tiempo, si un rasgo fenotípico de un organismo está generativamente atrincherado en su desarrollo, entonces hay otros rasgos del organismo que aparecieron durante su desarrollo ontogenético que son innatos (en nuestro sentido), siempre y cuando el rasgo original haya sido un factor causal de la aparición de estos otros rasgos junto con otros factores causales atrincherados en su desarrollo, entre los cuales se incluyen ciertos factores genéticos.

Ahora bien, si esto es correcto - si existen algunas relaciones interesantes entre mi noción de lo innato y la noción de atrincheramiento generativo

${ }^{27}$ La razón por la cual es probable que la fijación de un recuerdo particular no contaría como innata en un humano es que ésta requiere factores causales externos no típicos de ninguna población humana, p.ej., un estímulo sensorial específico. 
propuesta por Wimsatt-, entonces puedo usar esta ultima noción para formular una noción de grados de, por así decirlo, "innatez" en el espíritu de mi propuesta, explotando el hecho de que la noción wimsattiana de atrincheramiento es una noción de grados. Puedo, así, heredar algunas de las virtudes de su noción de atrincheramiento, su fertilidad teórica, sin recibir los problemas que tiene su caracterización de lo innato.

Un rasgo fenotípico $T$ de un organismo $O$ que pertenece a una población $P$ es innato en grado $n$ en $O$ en el tiempo $t$, si y sólo si

1. el organismo en cuestión posee $T$ en $t$;

2. $T$ apareció en $O$ durante su desarrollo ontogenético como resultado de ciertos factores causales entre los cuales se encuentran determinados factores ambientales, $C_{1}, \ldots$, $C_{m}$, típicos de la población $P$ con respecto al rasgo $T$ en $t$;

3. el promedio de los grados en los cuales cada uno de los factores $C_{i}(i=1, \ldots, m)$ está atrincherado en $O$ es $n$, y

4. algunos de los $C_{i}$ incluyen algunas formas de expresión genética.

Debo indicar que tanto la caracterización de lo innato que Wimsatt propone, como la que yo postulo son intentos por capturar, de maneras distintas, algún aspecto de la connotación evolucionista adaptacionista normalmente asociada a lo innato en las disciplinas cognoscitivas que aquí me interesan. Sin embargo, éstas difieren con respecto al grado de eficacia con el que capturan la connotación evolutivo-adaptativa antes mencionada. Por ejemplo, una consecuencia de la caracterización wimsattiana de lo innato es que, si un rasgo fenotípico o un factor ambiental $T$ es muy innato a un organismo $O$, entonces $T$ es un factor causal en la aparición en $O$ de muchos otros rasgos fenotípicos que han probado ser adaptativamente ventajosos para los miembros de la población a la que $O$ pertenece, pero no se sigue necesariamente que $T$ mismo sea adaptativamente ventajoso para $O$. En contraste, de acuerdo con mi caracterización de lo innato, si un rasgo fenotípico $T$ de un organismo $O$ es muy innato, entonces el rasgo $T$ es en sí mismo adaptativamente muy ventajoso para $O$; es decir, que la aparición de $T$ en los miembros de la población en cuestión es el resultado de la evolución por selección natural. Así, mi caracterización adopta de manera más directa y adecuada la connotación evolutivo-adaptativa de lo innato en los estudios cognoscitivos considerados.

Otra diferencia entre mi caracterización y la de Wimsatt consiste en que, para Wimsatt, un rasgo fenotípico $T$ de un organismo puede ser innato incluso cuando ningún factor genético de ningún tipo esté involucrado en la aparición de $T$ en ese organismo (y aun cuando ningún factor genético 
intervenga en los procesos de desarrollo en los que el rasgo $T$ es un factor causal y que culminan en los múltiples efectos adaptativos que determinan el grado de "innatez" de $T$ ), mientras que, en mi caracterización de lo innato, esto no es posible puesto que un requisito explícito de esta caracterización de lo innato es que haya al menos algunos factores causales genéticos involucrados en la aparición de cualquier rasgo innato, lo cual captura la parte menos objetable de la idea que a veces expresan los estudiosos del aprendizaje y la conducta en animales y humanos al hablar de lo innato como aquello que está "genéticamente especificado" (por ejemplo, Tooby y Cosmides 1990, p. 22).

Finalmente, la noción de lo innato que propongo explica algunas de las connotaciones asociadas a lo innato en la etología cognoscitiva que, de acuerdo con Wimsatt, su noción de atrincheramiento también puede explicar. La primera afirma que:

La conducta innata es relativamente resistente al cambio evolutivo.

De acuerdo con mi caracterización de lo innato, esta afirmación es plausible, puesto que, si la conducta de que se trate es muy innata (en mi sentido), entonces es adaptativa y es resultado de factores causales que están muy atrincherados en el organismo en cuestión, lo cual significa que es improbable que un cambio en esos factores causales origine un desarrollo posterior normal y un organismo viable.

La segunda connotación de lo innato en la etología cognoscitiva que recoge mi caracterización de lo innato es la siguiente:

Si ciertos rasgos innatos no aparecen durante el desarrollo, ocurrirán importantes defectos funcionales.

Si un rasgo es muy innato (en mi sentido), entonces es adaptativo y se debe a factores causales generativamente muy atrincherados, los cuales, entre más atrincherados estén, menos probable será que, en su ausencia, el desarrollo posterior sea normal y dé como resultado un organismo viable. Ahora bien, si un rasgo muy innato no aparece durante el desarrollo, entonces es probable que no hayan estado presentes algunos de los factores causales típicamente responsables de la aparición de ese rasgo; son factores causales muy atrincherados en el organismo en cuestion, y entre más atrincherados estén estos factores en ese organismo, más probable será que el resultado final del desarrollo incluya defectos funcionales importantes. Ergo, el que aparezcan algunos defectos funcionales importantes durante el desarrollo es un indicio de que son innatos algunos de los rasgos causalmente coasociados a los factores causales cuya ausencia redundó en aquellos defectos. 


\section{Conclusiones}

Revisé la literatura psicológica perteneciente a aquellas disciplinas que estudian el desarrollo cognoscitivo, el aprendizaje y el comportamiento en seres humanos y en otros animales desde un punto de vista evolutivo, y concluí que pueden detectarse dos connotaciones centrales en la manera como se usa el término "innato" en esas disciplinas: lo innato como aquello que es producto de la evolución por selección natural y lo innato como lo no aprendido. Después examiné y presenté de manera precisa tres propuestas revisionistas relativamente recientes para caracterizar una noción de lo innato usando conceptos e ideas de la biología y concluí que las primeras dos caracterizaciones de lo innato examinadas - la de Ariew y la de Wimsatt- son inadecuadas, puesto que tienen implicaciones que las alejan radicalmente de algunas de las connotaciones más importantes de lo innato tal y como se emplea el término en estas disciplinas. La tercera propuesta - la propia- no tiene los problemas de las anteriores y sí muchas de sus ventajas, además de que recoge de manera directa y precisa lo que llamamos la connotación evolucionista adaptacionista.

\section{BIBLIOGRAFÍA}

Ariew, A., 1999, "Innateness is Canalization: In Defense of a Developmental Account of Innateness", en Hardcastle 1999, pp. 117-138.

Bechtel, W. (comp.), 1986, Integrating Scientific Disciplines, Martinus Nijhoff, Dordrecht.

Carey, S. y R. Gelman (comps.), 1991, The Epigenesis of Mind: Essays on Biology and Cognition, Lawrence Erlbaum Associates Publishers, Hillsdale, Nueva Jersey.

Cosmides, L. y J. Tooby, 1998, "Evolutionizing the Cognitive Sciences: A Reply to Shapiro and Epstein", Mind and Language, vol. 13, no. 2, pp. 195-204.

_, 1997 , "The Modular Nature of Human Intelligence", en Scheibel y Schopf 1997, pp. 71-101.

_ 1994, "Origins of Domain Specificity: The Evolution of Functional Organization", en Hirschfeld y Gelman 1994, pp. 85-116.

Davies, P.S., 1996, "Preface: Evolutionary Theory in Cognitive Psychology", Minds and Machines, vol. 6, no. 4, pp. 445-462.

Elman, J.L., et al., 1996, Rethinking Innateness: A Connectionist Perspective on Development, The MIT Press, Cambridge, Mass.

Fodor, J., 1982a, "The Present Status of the Innateness Controversy", en Fodor 1982b, pp. 257-316.

— 1982b, Representations: Philosophical Essays on the Foundations of Cognitive Science, The MIT Press, Cambridge, Mass.

Futuyma, D.J., 1998, Evolutionary Biology, 3a. ed., Sinauer, Sunderland, Mass. 
Gallistel, C.R., A.L. Brown, S. Carey, R. Gelman y F.C. Keil, 1991, "Lessons from Animal Learning for the Study of Cognitive Development", en Carey y Gelman 1991, pp. 3-36.

García, Claudia Lorena, 2005, "Razonamiento, especificidad de dominio y sesgo en la psicología del razonamiento humano", Signos Filosóficos, en prensa.

—, 2004 , "Innatismo y biología: Hacia un concepto biológico de lo innato", Theoría (España), en prensa.

—_, 2003, "Biología e innatismo: Algunos comentarios críticos", Crítica, vol. 35, no. 104 , pp. 3-30.

Gerhart, J. y M. Kirschner, 1997, Cells, Embryos and Evolution: Toward a Cellular and Developmental Understanding of Phenotypic Variation and Evolutionary Adaptability, Blackwell Science, Malden, Mass.

Gigerenzer, G., 2001, “The Adaptive Toolbox", en Gigerenzer y Selten 2001, pp. 37-50.

Gigerenzer, Gerd y Reinhard Selten (comps.), 2001, Bounded Rationality: The Adaptive Toolbox, The MIT Press, Cambridge, Mass.

Griffiths, P.E. y R.D. Gray, 1994, "Developmental Systems and Evolutionary Explanation", Journal of Philosophy, vol. 91, no. 6, pp. 277-304.

Hardcastle, Valerie Gray (comp.), 1999, Where Biology Meets Psychology, The MIT Press, Cambridge, Mass.

Hirschfeld, L.A. y S.A. Gelman (comps.), 1994, Mapping the Mind: Domain Specificity in Cognition and Culture, Cambridge University Press, Cambridge.

Hull, D., 1999, "On the Plurality of Species: Questioning the Party Line", en Wilson 1999, pp. 23-48.

Kandel, E.R., J. H. Schwartz y T.M. Jessell, 1995, Essentials of Neural Science and Behavior, Appleton and Lange, Stamford, Conn.

Khalidi, M.A., 2002, "Nature and Nurture in Cognition", The British Journal for the Philosophy of Science, vol. 53, pp. 251-272.

Marler, P., 1991, “The Instinct to learn”, en Carey y Gelman 1991, pp. 37-66.

McClaurin, J., 2002, "The Resurrection of Innateness", The Monist, vol. 85, no. 1, pp. 105-130.

Newport, E. L., 1991, "Contrasting Conceptions of the Critical Period for Language," en Carey y Gelman 1991, pp. 111-130.

Samuels, R., 2002, "Nativism in Cognitive Science", Mind and Language, vol. 17, no. 3, pp. 233-265.

Scharloo, W., 1991, "Canalization: Genetic and Developmental Aspects", Annual Review of Ecology and Systematics, vol. 22, pp. 65-93.

Scheibel, A.B. y W.J. Schopf (comps.), 1997, The Origin and Evolution of Intelligence, Jones and Bartlett, Sudbury, Mass.

Shapiro, L. y W. Epstein, 1998, "Evolutionary Theory Meets Cognitive Psychology: A More Selective Perspective", Mind and Language, vol. 13, no. 2, pp. 171-194.

Shettleworth, S.J., 1998, Cognition, Evolution, and Behaviour, Oxford University Press, Oxford.

Sober, E., 1999, "Innate Knowledge", en The Routledge Encyclopedia of Philosophy, ed. E. Craig, Routledge, Londres/Nueva York, vol. 4, pp. 794-797.

Sperber, D., 1994, "The Modularity of Thought and the Epidemiology of Representations", en Hirschfeld y Gelman 1994, pp. 42-46. 
Tooby, J. y L. Cosmides, 1990, "On the Universality of Human Nature and the Uniqueness of the Individual: The Role of Genetics and Adaptation", Journal of Personality, vol. 58, no. 1, pp. 17-67.

Waddington, C.H., 1942, "The Canalization of Development and the Inheritance of Acquired Characters", Nature, vol. 150, pp. 563-565.

Wilson, R.A. (comp.), 1999, Species: New Interdisciplinary Essays, The MIT Press, Cambridge, Mass.

Wimsatt, W., 1986, "Developmental Constraints, Generative Entrenchment and the Innate-Acquired Distinction", en Bechtel 1986, pp. 185-208.

Recibido el 19 de agosto de 2003; aceptado el 1 de febrero de 2005. 